\title{
Severe acute respiratory syndrome coronavirus 2 vaccines: The good, the bad, and the ugly
}

\author{
Katia N. Estrada-Medrano ${ }^{1}$, Alejandro Rodríguez-Garza1, Mario C. Salinas-Carmona², and \\ David Gómez-Almaguer ${ }^{1 *}$ \\ ${ }^{1}$ Hematology Service, ${ }^{2}$ Department of Immunology, School of Medicine, University Hospital "Dr. José Eleuterio González", Universidad Autónoma \\ de Nuevo León, Monterrey, Nuevo Leon, Mexico
}

\begin{abstract}
The coronavirus disease pandemic is caused by the highly infectious severe acute respiratory syndrome coronavirus 2 (SARS-CoV-2). The infection can result in mild-to-severe disease, although many asymptomatic infections are described. There are many types of vaccines with different characteristics, and many have reached Phase III of clinical trials. In this review, we included the main vaccines approved worldwide, including those approved in Mexico. Vaccine-induced immune thrombotic thrombocytopenia has been described, especially in adenovirus vector vaccines. There are specific concerns around vaccine safety and efficacy in special populations such as pregnant women and immunosuppressed individuals. Even though the evidence is scarce, vaccine application remains recommended in most cases. We concluded that the risks of adverse effects from the vaccines are minimal compared to the current risk of contracting the SARS-COV-2 infection. The benefit of applying the vaccines is greater than not applying them at all.
\end{abstract}

Key words: Coronavirus disease. Vaccines. Vaccine development.

\section{Introduction}

At the end of 2019, a novel coronavirus now known as severe acute respiratory syndrome coronavirus (SARSCoV-2) was identified as the cause of a cluster of pneumonia cases in Wuhan, a city in China's Hubei Province. It rapidly spread, resulting in a global pandemic. SARSCoV-2 is now the leading cause of severe acute respiratory syndrome. As of May 2021, it has caused more than 154 million cases and over 3 million deaths worldwide'.

The dramatic increase in the virus spread, as well as raising the mortality rate of coronavirus disease (COVID-19) around the world, has led many researchers toward finding potential vaccine candidates for protection against SARS-CoV-2. An efficacious vaccine is essential to prevent further morbidity and mortality. Therefore, vaccine immunity has become a major public health challenge for governments, physicians, and scientists worldwide.

Vaccines against COVID-19 were an essential global intervention to control the current pandemic situation. Vaccines often cause adverse events; however, the vast majority of adverse events following immunization are a consequence of the vaccine stimulating a protective immune response and not allergic in etiology.

\section{Correspondence:}

*David Gómez-Almaguer

E-mail: dgomezalmaguer@gmail.com
Available online: $28-06-2021$

Date of reception: 07-05-2021

Date of acceptance: 12-05-2021 DOI: 10.24875/RMU.M21000057
Medicina Universitaria. 2021;23(2):70-73 www.medicinauniversitaria.org license (http://creativecommons.org/licenses/by-nc-nd/4.0/). 


\section{Overview of vaccine development}

The development of pharmaceuticals, vaccine development progresses through preclinical evaluation and three distinct clinical stages ${ }^{2}$ :

\section{Preclinical testing}

Scientists test a new vaccine on cells and then give it to animals such as mice or monkeys to see if it produces an immune response.

Phase I trials: These are designed to test vaccine safety, although immunogenicity is also measured; dose-ranging studies are also often included.

Phase II trials: These expand the safety profile and immune response assessment in a larger number of participants.

Phase III trials: These are designed to determine efficacy in preventing a predefined endpoint, usually laboratory-confirmed disease. The vaccine efficacy percentage is the reduction in disease incidence among those who received vaccination versus those who received the control product. Efficacy criteria for SARSCoV-2 trials are discussed elsewhere.

Conventionally, these steps occur sequentially, and each usually takes several years to complete. COVID-19 vaccine development has been accelerated, with each step occurring over several months. In addition, in the COVID-19 vaccine initiative, Phase I and II and Phase II and III studies have frequently been combined with a seamless transition from one phase into the next. At this moment, some vaccines are still in Phase III trials, such a Cansino, Sinovac, Gamaleya, Bharat Biotech, and their final Phase III results have not been published in any journal. However, some preliminary data have been published in their official press release(Table 1$)^{3-11}$.

\section{Vaccine-induced immune thrombotic thrombocytopenia (VITT)}

By the end of February, a thrombotic syndrome was observed in a small number of individuals who received the ChAdOx1 CoV-19 vaccine (AstraZeneca, University of Oxford, and Serum Institute of India), an adenoviral vector-based vaccine ${ }^{12}$. Afterward, similar findings were observed in a small number of individuals who received the Ad26.COV2.S vaccine (Janssen; Johnson and Johnson), also based on an adenoviral vector ${ }^{13}$. This syndrome has been designated VITT. It has also been called thrombosis with thrombocytopenia syndrome and vaccine-induced prothrombotic immune thrombocytopenia.

\section{Current vaccine recommendations for special populations}

\section{Allergy and anaphylaxis}

Contraindications to COVID-19 vaccination are severe or immediate allergic reactions to the vaccine or any of its components. All individuals should be monitored for an immediate reaction following vaccination. Individuals without a contraindication who have a history of anaphylaxis of any kind, an immediate allergic reaction to other vaccines or injectable therapies, or a contraindication to a COVID-19 vaccine class other than the one they are receiving should be monitored for $30 \mathrm{~min}$. All other individuals are monitored for $15 \mathrm{~min}$.

\section{Pregnant Women}

None of the vaccines listed in this review contain a virus that replicates; thus, they do not cause disease, but non-specific side effects from immune response activation may occur. Based on how mRNA and viral vector vaccines work, experts believe they are unlikely to pose a risk for pregnant persons, the fetus, or breastfeeding newborns ${ }^{14}$. It is advised for pregnant individuals to undergo COVID-19 vaccination as soon as it is available in their countries and avoid deferring until the end of pregnancy. Patients who are at higher risk of exposure to SARS-CoV-2 or at the highest risk of having the severe disease if infected (e.g. patients with diabetes, obesity, or heart disease) may benefit most. Data about the efficacy and safety of COVID vaccines in pregnant individuals are limited, but studies are underway ${ }^{15}$.

\section{IMMUNOSUPPRESSION}

Eligible individuals who have an immunocompromising condition or are taking immunosuppressive agents should undergo COVID-19 vaccination. Although the immunogenicity and efficacy of the vaccines are uncertain in such individuals and may be lower than those observed in the general population, the potential for severe COVID-19 in this population likely outweighs the uncertainties $^{16,17}$.

\section{IMMUNE RESPONSE IN COVID PATIENTS}

Individuals with a history of SARS-CoV-2 infection should still receive one of these vaccines. Individuals 


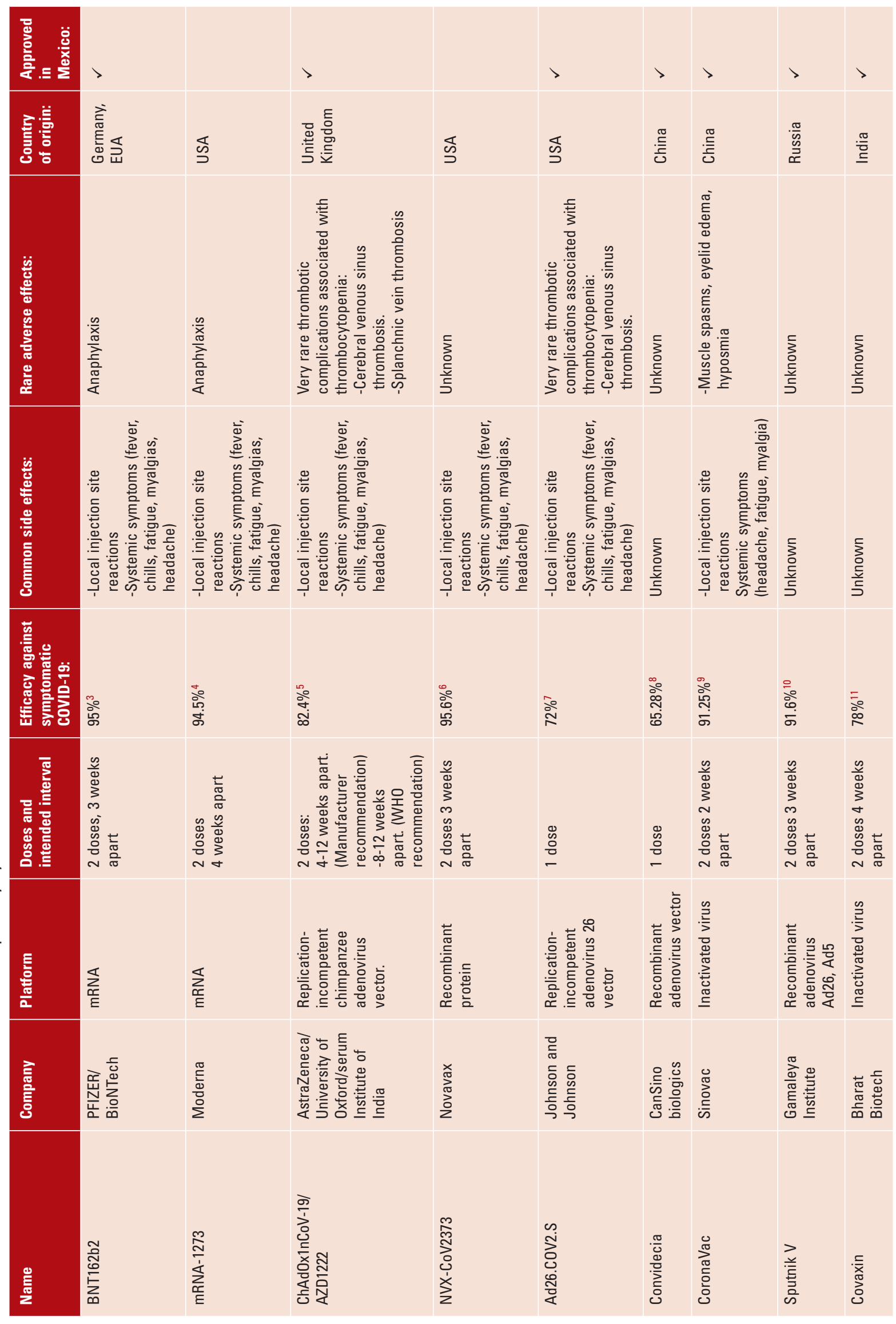


with recent, documented SARS-CoV-2 infection (including those diagnosed following the first vaccine dose) should have recovered from acute infection and met the criteria for discontinuation of isolation precautions before receiving the vaccine.

CD8 $T$ lymphocytes are critical for destroying virus-infected cells; their amount in peripheral blood is related to asymptomatic to mild and severe COVID-19 disease. It was recently published that SARS-CoV-2 specific $T$ lymphocytes play an important role in long-lasting protection and deserve further investigation after different vaccine applications ${ }^{18}$.

There is scientific evidence demonstrating that a single mRNA vaccine to individuals recovered from COVID-19 induces a robust anti-viral antibody response. However, antibody titer was no further increased after a second vaccination, showing that in recovered COVID-19 patients, one single mRNA vaccine boosts high neutralizing antibody titer and memory $B$ cells ${ }^{19}$.

\section{Conclusion}

Vaccine development, approval, and production have been accelerated to fight the COVID pandemic. Most of the available vaccines have not finished their Phase III trials but have been approved for emergency use. Nevertheless, the preliminary results indicate that all vaccines are safe, and we recommend the public not to hesitate or avoid any of the approved vaccines in their country. The current risks of adverse effects from the vaccines are minimum compared to the current risk of contracting the SARS-COV2 infection. Undesirable effects vary from local pain and skin rash to hyperthermia, irritation, and weakness. Still, all these are transitory and disappear in a day or 2 after inoculation with or without nonsteroidal anti-inflammatory drugs. Religious, philosophical prejudices, and misinformation in social media contribute to resistance to vaccination, but it is clear that benefits are by far superior to minor undesirable side effects ${ }^{20}$.

Even though it is important to remark the adverse reaction of prothrombotic syndrome observed in individuals who have received the ChAdOx1 CoV-2 vaccine (AstraZeneca, University of Oxford, and Serum Institute of India) and the Ad26.COV2., S vaccine (Janssen; Johnson and Johnson) would be worth the effort to do screening on a population that these reactions may develop and eventually choose which vaccine has a lower probability to cause these side effects. We consider, as mentioned before that the benefit of applying the vaccines is greater than not applying them at all. The economic impact of vaccination in all countries demonstrated through the years its utility. Therefore, there is not a bad or ugly vaccine.

\section{Conflicts of interest}

The authors declare that they have no conflicts of interest.

\section{Funding}

The authors declare that they have no funding.

\section{References}

1. World Health Organization. WHO Director-General's Remarks at the Media Briefing on 2019-nCoV. Geneva: World Health Organization; 2020. Available from: https://www.who.int/dg/speeches/detail/who-director-general-s-remarks-at-the-media-briefing-on-2019-ncov-on-11-february-2020.

2. Vaccine Development, Testing, and Regulation. 2014. Available from: https://www.historyofvaccines.org/content/articles/vaccine-development-testing-and-regulation.

3. Mahase E. Covid-19: pfizer vaccine efficacy was $52 \%$ after first dose and $95 \%$ after second dose, paper shows. BMJ. 2020;371:4826.

4. Mahase E. Covid-19: vaccine candidate may be more than $90 \%$ effective, interim results indicate. BMJ. 2020;371:m4347.

5. Wise J. Covid-19: new data on Oxford AstraZeneca vaccine backs 12 week dosing interval. BMJ. 2021;372:n326.

6. Mahase E. Covid-19: novavax vaccine efficacy is $86 \%$ against UK variant and $60 \%$ against South African variant. BMJ. 2021;372:n296.

7. Sadoff J, Gray G, Vandebosch A, Cárdenas V, Shukarev G, Grinsztejn B, et al. Safety and efficacy of single-dose Ad26.COV2.S vaccine against covid-19. N Engl J Med. 2021. doi: 10.1056/NEJMoa2101544. Epub ahead of print.

8. Mangi F, Lyu D, Ismail D. China's CanSino Vaccine Shows $65.7 \%$ Efficacy in One Shot-Bloomberg. Bloomberg. Available from: https://www. bloomberg.com/news/articles/2021-02-08/pakistan-says-cansino-s-covid-vaccine-shows-65-7-efficacy. [Last accessed on 2021 May 11].

9. Summary of Clinical Trial Data of Sinovac's COVID-19 Vaccine (CoronaVac®). Press Releases. Available from: http://www.sinovac.com/news/shownews.php?id=1154 and amp;lang=en. [Last accessed on 2021 May 11].

10. Jones I, Roy P. Sputnik V COVID-19 vaccine candidate appears safe and effective. Lancet. 2021;397:642-3.

11. Bharat Biotech-a Leading Biotech Company Press. Available from: https://www.bharatbiotech.com/press_releases.html. [Last accessed on 2021 May 11].

12. Greinacher A, Thiele T, Warkentin TE, Weisser K, Kyrle PA, Eichinger S. Thrombotic thrombocytopenia after ChAdOx1 nCov-19 vaccination. N Engl J Med. 2021;2021:1-10.

13. See I, Su JR, Lale A, Woo EJ, Guh AY, Shimabukuro T, et al. US case reports of cerebral venous sinus thrombosis with thrombocytopenia after Ad26. COV2.S vaccination, March 2 to April 21, 2021. JAMA. 2021;2021:e217517.

14. Male V. Are COVID-19 vaccines safe in pregnancy? Nat Rev Immunol. 2021;21:200-1.

15. Shimabukuro TT, Kim SY, Myers TR, Moro PL, Oduyebo T, Panagiotakopoulos $L$, et al. Preliminary findings of mRNA covid-19 vaccine safety in pregnant persons. N Engl J Med. 2021. doi: 10.1056/NEJMoa2104983. Epub ahead of print

16. Monin L, Laing AG, Muñoz-Ruiz M, Del Molino Del Barrio I, Alaguthurai T, Domingo-Vila $C$, et al. Safety and immunogenicity of one versus two doses of the COVID-19 vaccine BNT162b2 for patients with cancer: interim analysis of a prospective observational study. Lancet Oncol. 2021. doi.org/10.1016/ S1470-2045(21)00213-8. Epub ahead of print.

17. Herishanu Y, Aharon Sackler A, Tel Aviv SL, Tel Aviv MM. Efficacy of the BNT162b2 mRNA COVID-19 vaccine in patients with chronic lymphocytic leukemia. Blood. 2021. Available on: https://doi.org/10.1182/ blood.2021011568. Epub ahead of print.

18. Saini SK, Hersby DS, Tamhane T, Povlsen HR, Hernandez SP, Nielsen $\mathrm{M}$, et al. SARS-CoV-2 genome-wide T cell epitope mapping reveals immunodominance and substantial $\mathrm{CD}^{+} \mathrm{T}$ cell activation in $\mathrm{CO}$ VID-19 patients. Sci Immunol. 2021;6:eabf7550.

19. Goel RR, Apostolidis SA, Painter MM, Mathew D, Pattekar A, Kuthuru O, et al. Distinct antibody and memory $B$ cell responses in SARS-CoV-2 naïve and recovered individuals following mRNA vaccination. Sci Immunol. 2021;6:eabi6950.

20. Salinas-Carmona MC. Vaccination, an individual right, or a collective responsibility? Challenges and opportunities in the $21^{\text {st }}$ century. Rev Med Univ. 2019;21:38-9. 